\title{
The Increasing Dominance of Teams in Production of Knowledge
}

Stefan Wuchty, ${ }^{1 *}$ Benjamin F. Jones, ${ }^{2}{ }^{*}$ and Brian $\mathrm{Uzzi}^{3 * \dagger}$

${ }^{1}$ Northwestern Institute on Complexity (NICO), Northwestern University, Evanston, IL 60208, USA. ${ }^{2}$ Kellogg School of Management, Northwestern University, Evanston, IL 60208, USA. ${ }^{3}$ Kellogg School of Management, Northwestern University and the Northwestern Institute on Complexity (NICO), Evanston, IL 60208, USA (Corresponding author).

*Authors contributed equally to this work.

†To whom correspondence should be addressed. E-mail: uzzi@northwestern.edu

We have used 19.9 million papers over 5 decades and 2.1 million patents to demonstrate that teams increasingly dominate solo authors in the production of knowledge. Research is increasingly done in teams across virtually all fields. Teams typically produce more highly cited research than individuals do, and this advantage is increasing over time. Teams now also produce the exceptionally high impact research, even where that distinction was once the domain of solo authors. These results are detailed for the sciences and engineering, social sciences, arts and humanities, and patents, suggesting that the process of knowledge creation has fundamentally changed.

An acclaimed tradition in the history and sociology of science emphasizes the role of the individual genius in scientific discovery $(1,2)$. This tradition focuses on guiding contributions of solitary authors, such as Newton and Einstein, and can be seen broadly in the tendency to equate great ideas with particular names, such as the Heisenberg uncertainty principle, Euclidean geometry, Nash equilibrium, and Kantian ethics. The role of individual contributions is also celebrated through science's award-granting institutions, like the Nobel Prize Foundation (3).

Several studies, however, have explored an apparent shift in science from this individual-based model of scientific advance to a teamwork model. Building on classic work by Zuckerman and Merton, many authors have established a rising propensity for teamwork in samples of research fields, with some studies going back a century (4-7). For example, de Solla Price examined the change in team size in chemistry from 1910 to 1960, forecasting that in 1980 zero percent of the papers would be written by solo authors (8). Recently, Adams et al. established that teamwork had increased across broader sets of fields among elite U.S. research universities (9). Nevertheless, the breadth and depth of this projected shift in manpower remains indefinite particularly in fields where the size of experiments and capital investments remain small, raising the question as to whether the projected growth in teams is universal or cloistered in specialized fields.
A shift towards teams also raises new questions of whether teams produce better science. Teams may bring greater collective knowledge and effort, but they are known to experience social network and coordination losses that make them under-perform individuals even in highly complex tasks (10-12), as F. Scott Fitzgerald concisely observed when he stated that "no grand idea was ever born in a conference" (13). From this viewpoint, a shift to teamwork may be a costly phenomenon or one that promotes low-impact science, while the highest impact ideas remain the domain of great minds working alone.

We studied 19.9 million research articles in the Institute for Scientific Information (ISI) Web of Science database and an additional 2.1 million patent records. The Web of Science data covers research publications in science and engineering since 1955, the social sciences since 1956, and arts and the humanities since 1975. The patent data covers all U.S. registered patents since 1975 (14). A team was defined as having more than one listed author (publications) or inventor (patents). Following the ISI classification system, the universe of scientific publications is divided into three main branches and their constituent subfields: science and engineering (with 171 subfields), social sciences (with 54 subfields) and the arts and humanities (with 27 subfields). The universe of U.S. patents was treated as a separate category (with 36 subfields). See the Supplementary Material for details on these classifications.

For science and engineering, social sciences, and patents, there has been a substantial shift towards collective research. In the sciences, team size has grown steadily each year and nearly doubled from 1.9 to 3.5 authors per paper over 45 years.

Shifts toward teamwork in science and engineering have been suggested to follow from the increasing scale, complexity, and costs of big science. Surprisingly then, we find an equally strong trend towards teamwork in the social sciences, where these drivers are much less significant. Although social scientists in 1955 wrote $17.5 \%$ of their 
papers in teams, by 2000 they wrote $51.5 \%$ of their papers in teams, an increase similar to that in sciences and engineering. Mean team size has also grown each year. On average, today's social sciences papers are written in pairs with a continuing, positive trend towards larger teams. Unlike the other areas of research, single authors still produce over $90 \%$ of the papers in the arts and humanities. Nevertheless, there is a positive trend toward teams in the arts and humanities ( $p$ $<.001)$. Finally, patents also show a rising dominance of teams. Although this data is on a shorter time scale (19752000), there was a similar annualized increase in the propensity for teamwork. Average team size has risen from 1.7 to 2.3 inventors per patent, with the positive trend towards larger teams continuing.

The generality of the shift to teamwork is captured in Table 1. In sciences and engineering, 99.4\% of the 171 subfields have seen increased teamwork. Meanwhile, $100 \%$ of the 54 subfields in the social sciences, $85.2 \%$ of the 27 subfields in the humanities, and $100 \%$ of the 36 subfields in patenting have seen increased teamwork.

Supplementary table S1 presents trends for individual fields. In the sciences, areas like medicine, biology, and physics have seen at least a doubling in mean team size over the 45 year period. Surprisingly, even mathematics, long thought the domain of the loner scientist and least dependent of the hard sciences on lab scale and capital-intensive equipment, showed a marked increase in the fraction of work done in teams, from $19 \%$ to $57 \%$, with mean team size rising from 1.22 to 1.84 . In the social sciences, psychology, economics, and political science show enormous shifts toward teamwork, sometimes doubling or tripling the propensity for teamwork. With regard to average team size, psychology, the closest of the social sciences to a lab science, has the highest growth (75.1\%) while political science has the lowest (16.6\%). All areas of patents showed a positive change in both the fraction of papers done by teams and team size with only small variations across the areas of patenting, suggesting that the conditions favoring teamwork in patenting are largely similar across subfields. As reflected in Figure 1A, the humanities show lower growth rates in the fraction of publications done in teams, yet a tendency towards increased teamwork is still observed.

Our measure of impact was the number of citations each paper and patent receives, which has been shown to correlate with research quality (15-17) and is frequently used in promotion and funding reviews (18). Highly cited work was defined as receiving more than the mean number of citations for a given field and year (19). Teams produce more highly cited work in each broad area of research and at each point in time.

To explore the relationship between teamwork and impact in more detail, we define the relative team impact (RTI) for a given time period and field. RTI is the mean number of citations received by team-authored work divided by the mean number of citations received by solo-authored work. A RTI greater than 1 indicates that teams produce more highly cited papers than solo authors and vice versa for RTI less than 1 . When the RTI is equal to 1 , there is no difference in citation rates for team and solo authored papers. In our dataset the average RTI was greater than 1 at all points in time and in all broad research areas -- sciences and engineering, social sciences, humanities, and patents. In other words, there is a broad tendency for teams to produce more highly cited work than individual authors. Further, the RTI is rising with time. For example, in sciences and engineering, team-authored papers received 1.7 times as many citations as solo-authored papers in 1955, but 2.1 times the citations by 2000. Similar upward trends in relative team impact appear in sciences and engineering, social science, and arts and humanities, and more weakly in patents, although the trend is still upward there (20). Note especially that, during the early periods, solo authors received substantially more citations on average than teams in many subfields, especially within sciences and engineering (Fig. 2E) and social sciences (Fig. $2 \mathrm{~F}$ ). By the end of the period, however, there are virtually no subfields in sciences and engineering and social sciences where solo authors typically receive more citations than teams. Table S1 details the RTI for major individual research areas, indicating that teams currently have a nearly universal impact advantage. In a minority of cases the RTI declined with time (e.g. $-34.4 \%$ in mathematics and $-25.7 \%$ in education), although even here teams currently have a large advantage in citations received (e.g. 67\% more average citations in mathematics and $105 \%$ in education).

The citation advantage of teams is also increasing with time when teams of fixed size are compared to solo authors. In Science and Engineering, for example, papers with two authors received 1.30 times more citations than solo authors in the 1950s but 1.74 times more citations in the 1990s. In general, this pattern prevails for comparisons between teams of any fixed size versus solo authors (table S4).

A possible challenge to the validity of these observations is the presence of self-citations given that teams have the opportunity to self-cite their work more frequently than a single author. To address this, we reran the analysis with all self-citations removed from the dataset (21). We found that removing self-citations can produce modest decreases in the RTI measure in some fields; for example, the RTI fell from 3.10 to 2.87 in Medicine and 2.30 to 2.13 in biology (table S1). Thus, removing self-citations can reduce the RTI by 5$10 \%$, but the relative citation advantage of teams remains essentially intact.

Because the progress of knowledge may be driven by a small number of key insights (22), we further test whether the 
most extraordinary concepts, results, or technologies are the province of solitary scientists or teams. Pooling all papers and patents within the four research areas, we calculated the frequency distribution of citations to solo-authored and teamauthored work, comparing the first five years and last five years of our data. If these distributions overlap in their right hand tails, then a solo-authored paper or patent is just as likely as a team-authored paper or patent to be extraordinarily highly cited.

Our results show that teams now dominate the top of the citation distribution in all four research domains (Fig 3, A-D). In the early years, a solo author in science and engineering or the social sciences was more likely than a team to receive no citations, but a solo author was also more likely to garner the highest number of citations - to be a paper that was singularly influential. However, by the most recent period, a teamauthored paper has a higher probability of being extremely highly cited. For example, a team-authored paper in science and engineering is currently 6.3 times more likely than a soloauthored paper to receive at least 1,000 citations. Finally, in the arts and humanities and patents, individuals were never more likely than teams to produce the more influential work. These patterns also hold when self-citations are removed (fig. S5).

Taken together, these results suggest two important facts about preeminent work in our observational periods. One, it never appeared to be the domain of solo authors in the arts and humanities and patents. Second, solo authors did produce the papers of singular distinction in science and engineering and social science in the 1950s, but the mantel of extraordinarily cited work has passed to teams by 2000 .

Over our 5 decade sample period, the increasing capital intensity of research may have been a key force in laboratory sciences where the growth in teamwork has been intensive (8), but it is unlikely to explain similar patterns in mathematics, economics, and sociology where we found that growth rates in team size have been nearly as large. Since the 1950 's the number of researchers has grown as well, which could promote finer divisions of labor and more collaboration. Similarly, steady growth in knowledge may have driven scholars toward more specialization, prompting larger and more diverse teams $(7,10)$. However, we found that teamwork is growing nearly as fast in fields where the number of researchers has grown relatively slowly (see Supplementary Material). Declines in communication costs could make teamwork less costly as well $(9,25)$. Shifting authorship norms may have influenced coauthorship trends in fields with extremely large teams, such as biomedicine and high-energy physics $(26,27)$, and yet our results hold across diverse fields where norms for order of authorship, existence of post doctorates, and prevalence of grant-based research differ substantially.

\section{References and Notes}

1. R. K. Merton, Science 159, 56 (1968).

2. P. J. Bowler, I. R. Morus, Making Modern Science: A Historical Survey (University of Chicago, Chicago, 2005).

3. J. F. English, The Economy of Prestige: Prizes, Awards, and the Circulation of Cultural Value (Harvard, Cambridge, MA, 2005).

4. R. Collins, The Sociology of Philosophies: A Global Theory of Intellectual Change (Harvard University Press, Cambridge, MA, 1998).

5. D. Cronin, D. Shaw, K. La Barre, Journal of the American Society for Information Science and Technology, 54, 855 (2003).

6. H. Zuckerman, R. K. Merton in The Sociology of Science, R. K. Merton, N. Storer Eds. (Univ. of Chicago Press, Chicago, 1973), pp. 545-550.

7. B. F. Jones, National Bureau of Economic Research Working Paper \#11360 (2005).

8. D. J. de Solla Price, Little Science, Big Science (Columbia, New York, 1963).

9. J.J. Adams, G. Black, R. Clemmons, P. E. Stephan, Research Policy 34, 259 (2005).

10. R. Guimerà, B. Uzzi, J. Spiro, L. Amaral, Science 308, 697 (2005).

11. N. Babchuk, K. Bruce, P. George, The American Sociologist 30, 5 (1999).

12. K. Y. Williams, C. A. O'Reilly in Research in Organizational Behavior, B. Staw and Robert Sutton, Eds. (JAI Press, Greenwich, CT, 1998), vol. 20, pp. 77-140.

13. F. S. Fitzgerald, in The Crack Up, E. Wilson, Ed. (New Directions, New York, 1993) 122.

14. B.H. Hall, A.B. Jaffe, M. Trajtenberg, National Bureau of Economic Research Working Paper \#8498 (2001).

15. M. Trajtenberg, RAND Journal of Economics, 21, 172 (1990).

16. B.H. Hall, A.B. Jaffe, M. Trajtenberg, RAND Journal of Economics, 36, 16 (2005).

17. D. W. Aksnes, Journal of the American Society for Information Science and Technology 57, 169 (2006).

18. N. S. Ali, H. C. Young, N. M. Ali, Library Review 45, 39 (1996).

19. Citations received were counted from publication year to 2006. Recent publications have smaller citation counts because they have had less time to be cited, but this effect is standardized when comparing team versus solo publications within a given year.

20. In patenting, we may observe weaker trends because citing earlier work can limit a patent's scope, so that applicants may avoid citations and (b) patent examiners typically add the majority of citations, which makes patent citations different from paper citations $(23,24)$. 
21. A self-citation is defined as any citation where a common name exists in the authorship of both the cited and citing papers. All citations were removed in which a citing and cited author's first initial and last name matched. Note that this method can also eliminate citations where the authors are different people but share the same name. However, performing Monte Carlo simulations on the data, we find that such errors occur in less than 1 of every 2,000 citations. Thus, any errors introduced by this method appear negligible. We did not remove self-citations from patents because citations to previous work in the patent literature are primarily assigned by the patent examiner (24), who independently assigns citations to earlier work based on the relevance of previous patents' content.

22. T. S. Kuhn, The Structure of Scientific Revolutions (University of Chicago, Chicago, 1970).

23. B. Sampat, "Determinants of Patent Quality: An Empirical Analysis”, mimeo, Columbia University, 2005.

24. J. Alcacer, M. Gittelman, Review of Economics and

Statistics 88, 774 (2006).

25. G. Becker, K. Murphy, Quarterly Journal of Economics 107, 1137 (1992).

26. J. Drenth, Journal of the American Medical Association 280, 219 (1998).

27. B. Cronin, Journal of the American Society for Information Science and Technology 52, 558 (2001).

28. We thank R. Guimera, S. Stern, K. Murnighan, and K. Williams Phillips, and two anonymous referees for their helpful comments. The Northwestern Institute on Complex Systems provided financial support.

\section{Supporting Online Material}

www.sciencemag.org/cgi/content/full/1136099/DC1

SOM Text

Figs S1 to S5

Tables S1 to S5

References

10 October 2006; accepted 3 April 2007

Published online 12 April 2007; 10.1126/science.1136099

Include this information when citing this paper.

Fig. 1. The growth of teams. These plots present changes over time in the fraction of papers and patents written in teams (panel A) and in mean team size (panel B). Each line represents the arithmetic average taken over all subfields in each year.

Fig. 2. The relative impact of teams. Panels A-D present mean team size comparing all papers and patents with those that received more citations than average in the relevant subfield. Panels E-H plot the "relative team impact” (RTI), which is the mean number of citations received by team- authored work divided by the mean number of citations received by solo-authored work. A ratio of 1 indicates that team and solo-authored work have equivalent impact on average. Each point in the plots represents the RTI for a given subfield and year, while the black lines present the arithmetic average in a given year.

Fig. 3. Exceptional research. Pooling all publications and patents within the four research categories, we calculated frequency distributions of citations received. Separate distributions are calculated for single authors and teams, and the ratio is plotted. A ratio greater than 1 indicates that a team-authored paper had a higher probability of producing the given range of citations than a solo-authored paper. Ratios are compared for the early period (first 5 years of available data) and late period (last 5 years of available data) for each research category, sciences and engineering (A), social sciences (B), arts and humanities (C), and patents (D). 
Table 1. Patterns by subfield. For the three broad ISI categories and for patents, we count the number $(\mathrm{N})$ and percentage (\%) of subfields that show (1) larger team sizes in the last five years compared to the first five years and (2) relative team impact measures (RTI) larger than 1 in the last five years. We show the RTI measures both with and without self-citations removed in calculating the citations received.

\begin{tabular}{|c|c|c|c|c|c|c|c|}
\hline \multirow{2}{*}{ fields } & \multirow{2}{*}{$\mathrm{N}_{\text {fields }}$} & \multicolumn{2}{|c|}{$\begin{array}{l}\text { increasing } \\
\text { team size }\end{array}$} & \multicolumn{2}{|c|}{$\begin{array}{l}\text { RTI > } 1 \\
\text { (with self citations) }\end{array}$} & \multicolumn{2}{|c|}{$\begin{array}{l}\text { RTI }>1 \\
\text { (no self citations) }\end{array}$} \\
\hline & & $\mathrm{N}_{\text {fields }}$ & $\%$ & $\mathrm{~N}_{\text {fields }}$ & $\%$ & $\mathrm{~N}_{\text {fields }}$ & $\%$ \\
\hline Science \& Engineering & 171 & 170 & 99.4 & 167 & 97.7 & 159 & 92.4 \\
\hline Social Sciences & 54 & 54 & 100.0 & 54 & 100.0 & 51 & 94.4 \\
\hline Arts \& Humanities & 27 & 24 & 88.9 & 23 & 85.2 & 18 & 66.7 \\
\hline Patents & 36 & 36 & 100.0 & 32 & 88.9 & -- & -- \\
\hline
\end{tabular}


Science

\&

Engineering
Social

Sciences
Arts

\&

Humanities
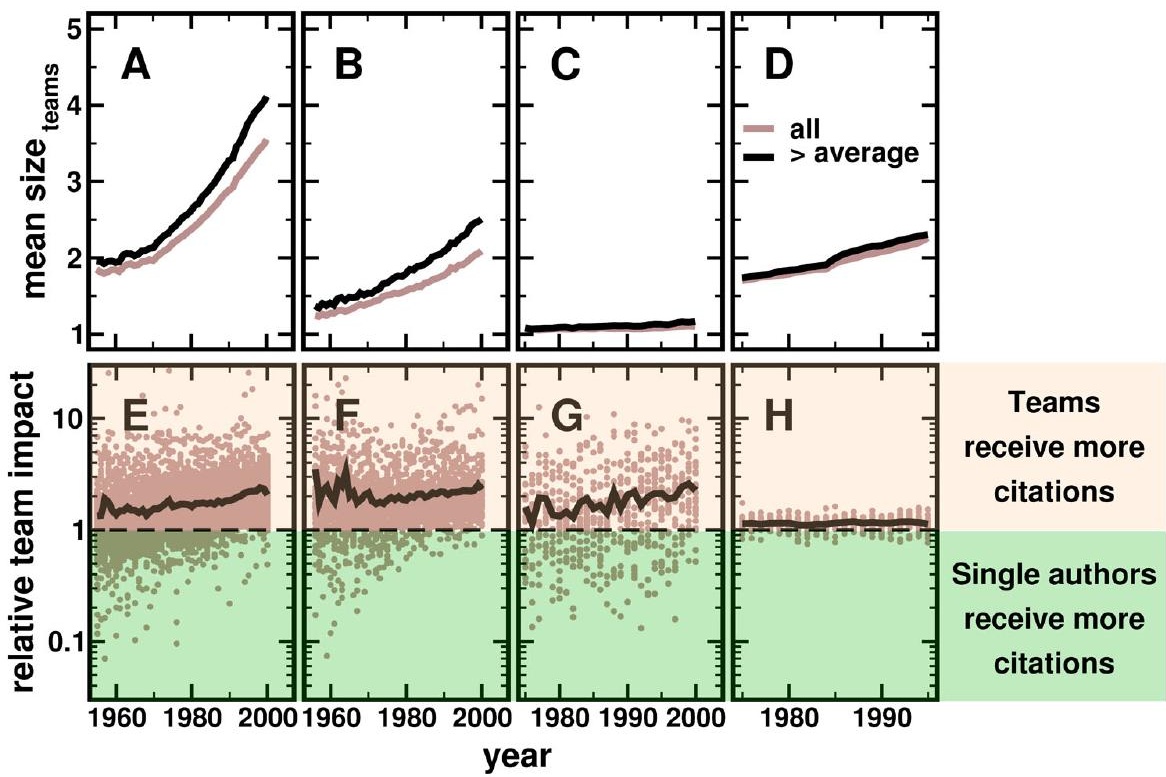


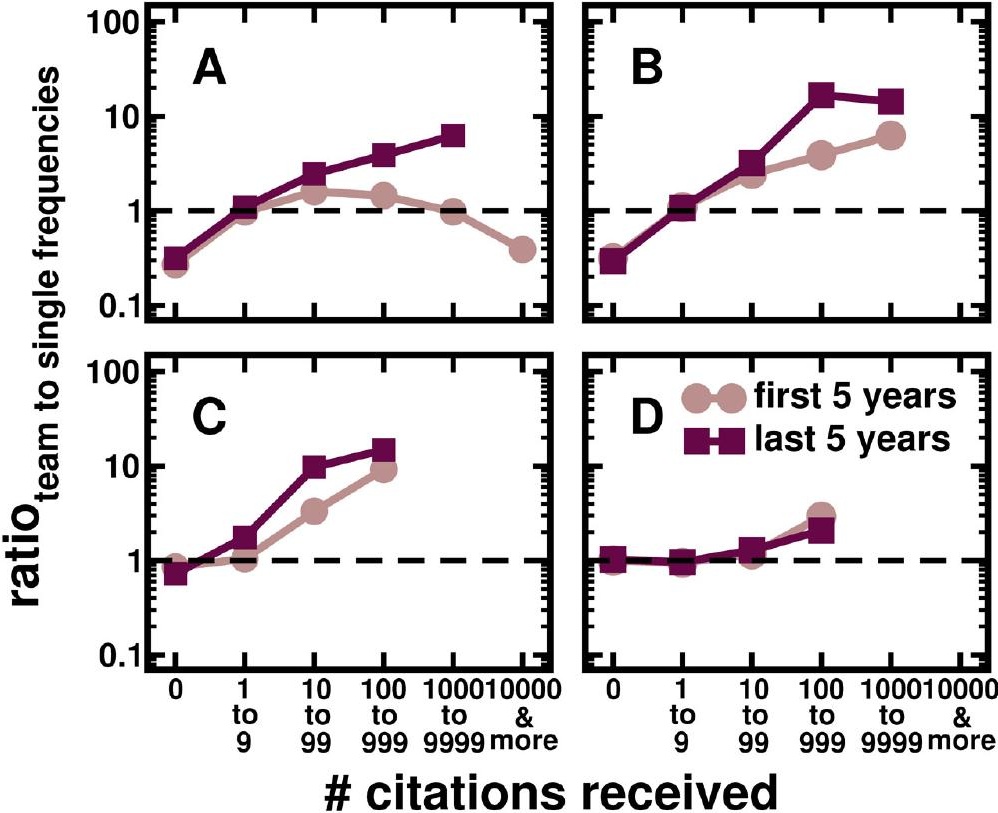

